\title{
Response of a Coriolis Mass Flow Meter to Step Changes in Flow Rate
}

\author{
Manus Henry, Mihaela Duta, Michael Tombs, University of Oxford, \\ Colin Clark, Robert Cheesewright, Brunel University
}

\begin{abstract}
Trials have taken place to determine the response of a prototype Coriolis mass flowmeter to step changes in flow rate. The meter typically exhibits a delay of $16 \mathrm{~ms}$, and tracks step changes well. Comparison with previously published results suggests that this performance represents an improvement over several types of current commercial flowmeters. The effect of a correction technique to reduce noise is demonstrated. This is considered valuable, even though it is responsible for $6 \mathrm{~ms}$ of the overall delay. A good dynamic response is important in process industry applications with short batch times.
\end{abstract}

\section{Introduction}

Coriolis mass flow metering is a well-established method of industrial flow measurement. The basic measurement principle is that a flowtube is caused to vibrate sinusoidally at a resonant frequency by one or more drivers, while two sensors monitor the vibration. The flowtube geometry and sensor placement are arranged so that the frequency of oscillation (which may vary from $80 \mathrm{~Hz}$ to $800 \mathrm{~Hz}$ for different flowtubes) can be used to calculate the density of the process fluid, while the phase difference between the two sensor signals provides the mass flow rate. The primary benefit of Coriolis metering is the direct measurement of mass flow, which is important where commodity value is related to mass rather than volume, for example in the petrochemical industry. However, Coriolis meters have other advantages, including high accuracy (to $0.1 \%$ ), and turndown (100:1 or better), while their limitations include the need for a separate power supply and relative expense.

As many industries move towards more flexible manufacturing practices, interest is increasing in the development of short, batch-oriented processes as opposed to continuous, steady operation. For optimal process management and reduced start-up times there is a need for flowmeters capable of measuring dynamic flows accurately. Brunel University is carrying out a research program to investigate the dynamic response of Coriolis flowmeters which includes; (i) a theoretical analysis of the response of a straight tube meter; (ii) simulation, by finite element analysis, of the dynamic response of more complex geometry meters; and (iii) an experimental investigation of the response of commercially available flowmeters to both step changes in flow rate and low frequency (relative to the meter drive frequency) pulsations. The University of Oxford, as part of its research program into self-validating sensors, has developed a novel mass flowmeter transmitter, capable of driving several different commercial flowtubes, based entirely on digital technology. Trials have been carried out on the Oxford prototype meter to measure its dynamic response to step changes in flow using the Brunel test facility. A recent study [1] reported on the dynamic response of a number of different commercial flowmeter technologies. A comparison with the results from the prototype meter is given at the end of this paper.

The paper is set out as follows. Firstly, the issue of the dynamic response of a Coriolis mass flow meter is discussed. Secondly, the features of the Oxford prototype are outlined. The experimental apparatus and procedure are described, and are followed by the results obtained and their analysis. In the final section, the implications for industrial applications are considered.

\section{Dynamic Response of Coriolis Mass Flow Meters}

The static repeatability and accuracy of mass flowmeters have been established for many years. There has been a steady increase in the market for these meter types as they replace older, less accurate metering technology. However, as industry moves towards more flexible manufacturing processes, with short batch times and rapid turnaround, the question of the dynamic response of flowmeters becomes more important. Specifically, given the excellent steady-state flow performance of Coriolis meters, is this matched by the dynamic response to flow changes? Potential applications for meters 
with a fast response time include short batch processes, and monitoring the response of safety valves during shutdown. Other potential benefits of responsive flowmeters are described by Wiklund and Peluso [2].

The response of the meter tube(s) to a step change in flow rate cannot be obtained over a period of less than one complete cycle of the driven motion because this is the shortest period over which a meaningful measurement of the phase difference between the sensor signals can be made (estimates over any shorter period would require an a priori knowledge of the exact shape of the sensor signal waveform, which is not available). It should be noted that this limitation on the response of the meter is not relaxed when the phase difference algorithm outputs two (or more) estimates of the phase difference per drive cycle, because each estimate is taken over one complete drive cycle (i.e. successive estimates are not independent). One implication of this restriction on the meter response is, for example, that a flow step which takes place in 5 milliseconds would be completed within one drive cycle of a meter driven at $100 \mathrm{~Hz}$, but would allow five separate estimates of flow throughout the step from a meter driven at $1 \mathrm{kHz}$.

For any practical Coriolis application, there are a number of additional stages that must be considered in assessing the overall dynamic response of the meter. These are illustrated in fig.1. The flowtube consists of the flow piping, the sensors and driver(s) together with housing and wiring. Conventionally the flowtube is viewed as separate from the transmitter, which processes the sensor data to generate measurement information and also generates the drive signal(s) to maintain flowtube oscillation. Within the transmitter some form of front-end circuitry is required as well as the processor, which performs flowtube control calculations and generates measurement data (typically temperature, density and mass flow) in engineering units. Each stage introduces additional delays due to processing time and, possibly, filtering. Because the transmitter processor is embedded, in practice its measurement data are not readily accessible to the outside world, and therefore a mechanism to transmit this data is required in the form of output circuitry. Finally, the monitoring system (whether an industrial control system or a laboratory data acquisition system) must have its own sampling circuitry to acquire the output signal, before taking actions such as executing a control algorithm or appending to a data record.

Any practical assessment of the dynamic response of a meter must consider all delays in the chain of events starting from a physical step change in flow within the pipework all the way through to this step change being registered by the processor in the monitoring system. This delay can be monitored experimentally from the vantage point of Observation 1 in fig. 1.

At the next stage, the sensor data are acquired in digital form within the transmitter, which entails analog-to-digital (ADC) conversion and, in some transmitters, an additional filtering stage. The processor calculations may introduce significant delay, as typically these calculations are not carried out continuously, but only once, or at most a few times, per drive cycle. It is possible to identify a further two stages within this delay. Firstly, sufficient measurement data are accumulated to perform the calculation (say during one complete cycle), then the calculation itself takes place. Where intensive calculations are used, it is computationally optimal for such calculations to take as long as the data collection period, and for the two operations to carry on in parallel. Thus, one drive cycle may be required to collect data, then a further drive cycle to process it, leading to an overall delay of 2 drive cycles between the first data of a step change being read by the ADC and the corresponding change appearing in the measurement data calculated by the processor.

The result of the transmitter processor calculations is the first representation of the flow measurement in engineering units. However fast the communications to the monitoring system, it can only add delay to the dynamic response of the measurement as seen by the transmitter processor. It is thus valuable for the purposes of evaluation to have direct access to the measurement values generated by the transmitter processor. For commercial systems this is not usually possible, but in the Oxford prototype system the mass flow values, which are calculated every half cycle, can be recorded and stored for analysis. This provides data from the vantage point of Observation 2 in fig. 1. 
In most industrial applications, the most important yardstick of dynamic response is the time taken for the control system to observe a change in flow, i.e. viewed from Observation 3 in fig. 1 . When considering the communication of data from the transmitter processor to the monitoring system, via whichever means (as described shortly), there are two basic decisions that must be taken. Firstly, how often a measurement update will be provided to the output circuitry, and secondly how the measurement update value will be calculated from the measurement data generated by the transmitter processor. It is of course possible to provide a measurement update every time a new measurement value is calculated, typically one or more times per drive cycle. However, given the conventional scanning rates of industrial control systems, it is more typical for updates to be provided at a rate of $10 \mathrm{~Hz}$ or slower. Given this update rate, the most accurate representation of the measurement data over the last (say) $100 \mathrm{~ms}$ would be to provide its average as the measurement update value. This introduces on average (say) 50ms delay in the response of the flowmeter. However, it is common to introduce additional filtering at this stage, in order to smooth the reported measurement value. With time constants of typically 100-1000 ms, such filtering can be the most significant influence in the dynamic response of commercial meters.

Presently there are three commonly-used industrial communication protocols:

- 4-20mA. With this protocol, the flow rate is mapped onto an analog current signal between 4 and $20 \mathrm{~mA}$. Being continuous, there is no delay in propagating the signal to the monitoring system, but there can be delay and filtering in the analog current circuitry. Furthermore, in the monitoring system, the signal is sampled using an ADC, which in the process control industry typically operates at $10 \mathrm{~Hz}$ or slower, leading to a further $50 \mathrm{~ms}$ or more average delay before the measurement is received by the monitoring processor. A major limitation of $4-20 \mathrm{~mA}$ is that it can introduce significant additional noise to the measurement signal through the need for in-situ calibration, analog circuit drift and ADC precision errors. This continuous, analog protocol has been dominant in the process industries over the last two decades, but is now giving way to various forms of digital communications.

- Pulse (frequency) output. This consists of a square wave signal in which the frequency of the pulse stream gives an indication of the instantaneous flowrate. This has some of the advantages of 4-20mA, being simple, unidirectional and continuous, while the discrete signal edges give some benefits of digital transmission, including higher precision. There are delays inherent in the means of communication, however. Typically the upper limit on the output is about $10 \mathrm{kHz}$, as higher frequencies may not be successfully propagated in a noisy industrial environment. It is not uncommon for zero flow to be mapped onto zero $\mathrm{Hz}$, so that at low flowrates there can be nontrivial delays in propagation due to the timing between edges - for example at $200 \mathrm{~Hz}$ there is a $5 \mathrm{~ms}$ period between rising edges. If the pulse output frequency is only updated after each rising edge (say), then this can lead to several milliseconds delay in propagating a step change from a low to a high flow value.

- Fieldbus communications (including HART). Various digital communication protocols allow the transmission of measurement data in floating point format, with no loss of precision. This is achieved through the transmission of discrete data packets. Again, typically in the process industries, measurement data is transmitted no more frequently that every $100 \mathrm{~ms}$, which places a severe restriction on the ability to assess the dynamic response of the meters.

It is likely that in the future, higher-speed digital communication protocols, such as industrial ethernet, will permit the transmission of the measurement data as seen by the transmitter processor directly to the monitoring processor with no loss of precision and with minimal delay (e.g. < 1ms). Given the current state of technology, however, the means selected to monitor the dynamic response of the Coriolis meters in the Brunel trials has been via the pulse output. This provides a continuous measurement signal, with higher precision than 4-20mA and without the inherent temporal discontinuities of fieldbus protocols, thus providing the best opportunity for transmitters to demonstrate a good dynamic response to step changes in flow rate. 


\section{The Oxford Prototype transmitter}

The Sensor Validation Research Group at the University of Oxford has been investigating the concept of the Self-Validating (SEVA) sensor for the last 15 years [3]. SEVA now forms the basis of a British Standard for reporting of measurement quality in industry [4]. A number of prototype sensors have been developed to demonstrate the feasibility of SEVA, of which the Coriolis meter is the most developed. Much of the background to the Coriolis meter transmitter developed at Oxford is described in [5]. However, further improvements have been made. The rapid pace of digital technology has afforded a simplification of the architecture of the transmitter, while at the same time improving the measurement performance that is achieved. For example, [5] reports that the standard deviation of the phase difference measurement for an empty flowtube is 1.8e-3 degrees; with the new transmitter this has been reduced to $2.1 \mathrm{e}-4$ degrees.

The architecture has been considerably simplified from that described in [5]. The main components are now a single processor, a single Field Programmable Gate Array (FPGA) and a codec (figure 2). The processor is a Pentium MMX 266MHz running the VxWorks real-time operating system. The FPGA is a Xilinx Spartan IIE-300, providing approximately 300,000 gates of configurable logic. This device carries out all real-time i/o, including the synthesis of the drive waveforms and generation of pulse outputs. The FPGA functionality has been defined using the Handel-C language [6,7]. The codec is designed for use in audio applications, providing two channels of input and two of output. These are used to monitor the sensor signals and to supply the drive output. Both input and output channels operate at $40 \mathrm{kHz}$ with 24 bit precision.

In the trials described in this paper, the B-shaped Coriolis flowtube described in [5] has been used, which typically is driven at around $80 \mathrm{~Hz}$, This design is unusual in using two drivers. However, the transmitter has successfully been used to operate other flowtube designs, including straight tube geometries, with drive frequencies of up to $400 \mathrm{~Hz}$. When operating a flowtube with only a single driver, the second drive output channel provided by the transmitter is simply not connected.

The transmitter provides up to five pulse output channels. The use of an FPGA allows high precision output to be generated [8]. This, together with the inherently high precision of the measurement calculation (see below) means that it is possible to provide an acceptably smooth pulse output signal even when measurement updates are provided every half cycle. This is important for the purposes of the dynamic response of the meter, as the large time constants associated with slow update rates or output filtering, of the order of 100-1000ms, are thus avoided.

It is possible to estimate the delays built into the prototype transmitter in the terms of the last section:

- Although the codec samples at about $40 \mathrm{kHz}$, there is a 61 sample 'group delay' i.e. there are 61 samples delay between input and output. This corresponds to approximately $1.5 \mathrm{~ms}$ delay for ADC sampling.

- Further pre-filtering takes place within the FPGA, creating a further 1ms delay.

a The drive frequency of a $25 \mathrm{~mm}$ flowtube filled with water is typically $82.4 \mathrm{~Hz}$. The processor performs measurement calculations every half cycle, at $165 \mathrm{~Hz}$ or every $6 \mathrm{~ms}$. However, a further half-cycle of data is required to perform an important measurement correction (as discussed below), so in total there is a delay of approximately $12 \mathrm{~ms}$ for data acquisition. The processor required a further $1.5 \mathrm{~ms}$ to perform the measurement calculation.

- The pulse output is updated immediately after the measurement calculation has been completed, and there are negligible delays $(<1 \mathrm{~ms})$ in propagating a step change in flowrate through to the pulse output, even for low flowrates.

This analysis suggests a total delay of approximately $16 \mathrm{~ms}$ from sensor signal input through to pulse output.

\section{Brunel Experimental Test Facility}


The experimental water flow test rig is shown in fig. 3. Flow is produced by a constant speed positive displacement pump and, by the use of by-passes, the flowrate through the test meter can be set in the range 0.3 to $8 \mathrm{~kg} / \mathrm{s}$. Outflow is to a weigh-tank which provides the primary flowrate reference measurement to an uncertainty of $\pm 0.1 \%$. The weigh-tank then discharges to a sump.

Two methods are providing for generating step changes in flowrate. In the first, a variable-area orifice plate is moved at high speed across the flow, either increasing or decreasing the orifice area and hence the flowrate; the mechanism is located just downstream of the Coriolis flowmeter. This method allows for relatively large step changes in flow e.g. from 0.2 to $0.8 \mathrm{~kg} / \mathrm{s}$ and, also, the fastest step change which is within $4 \mathrm{~ms}$. However, the orifice plate device generates considerable mechanical vibration in producing the step change.

The second method uses the sudden opening of a by-pass line to produce smaller step reductions in flowrate through the meter. This is achieved by closing off the end of the liquid filled by-pass (in parallel with the Coriolis meter) with a thin stretched plastic diaphram which is burst by a rapid burning of the membrane. This is achieved by the sudden discharge of electrical energy through a high resistance electrical coil in contact with the diaphragm. This mechanism produces slow steps (approximately 100ms) with very low levels of vibration.

Additional instrumentation is provided to enable full characterization of the dynamic features of the flow step. A commercial electromagnetic flowmeter provides a clear indication of the time-course of step. This is achieved by the use of continuous dc excitation of the meter which allows an extremely good dynamic response, but at the expense of a poor steady-state response, as a result of polarization effects at the sensor electrodes (these polarization effects were found to be negligibly small over the period of the steps used in this work). A pressure transducer is located just upstream of the two flowmeters and accelerometers are mounted adjacent to the flow step mechanisms. The accelerometer signal has a small dc component and the first zero-crossing is used to trigger the collection of data and for time synchronization between the two computers used for data logging.

The first computer logs four channels of data, these being from the pressure sensor, accelerometer, electromagnetic flowmeter and the pulse output from the Coriolis meter (Observation 3); the data sampling rate is typically $51.2 \mathrm{kHz}$. The second computer logs the two Coriolis sensor signals at a sampling rate of $500 \mathrm{kHz}$; these data are processed separately and are used to verify independently the change in phase difference as a result of the step change in flow (Observation 1). Finally, the Coriolis meter system itself records phase difference data every half-cycle (Observation 2).

\section{Results}

Figures 4 and 5 show the observed pulse output after a rapid $(4 \mathrm{~ms})$ and slower $(105 \mathrm{~ms})$ step changes in massflow. Both results were obtained using the variable area orifice plate method. The electromagnetic flowmeter signal is also shown to provide the reference time-history for the massflow step. Note that these signals are collected simultaneously by the Brunel test equipment, and thus there is no issue of time synchronization between them. In each figure the vertical dashed line shows the internally calculated transmitter delay, typically $16 \mathrm{~ms}$, after the step change begins. This transmitter delay is found by time-stamping ADC data as it is collected, and calculating the delay between real time and the timestamp of the ADC data when it has been processed to generate a flow measurement. In each figure the pulse output responds to the flow step change around the time predicted by the transmitter delay, thus validating the delay calculation.

Figures 6 and 7 show the massflow value (for the same experiments shown in figs. 4 and 5) as calculated by the transmitter's processor and the pulse output placed on the same timescale. This compares the mass flow measurements as seen from Observations 2 and 3 from fig. 1. Updates are generated every half-cycle of the drive frequency, or at about $6 \mathrm{~ms}$. The pulse output is clearly a good representation of the processor-calculated value. The dithering of the pulse output signal is a technique used to ensure that the average frequency over a reasonable timescale (e.g. 1s) is accurate to 1 part in 10 million [8]. In reality, the transmitter processor value is calculated before the pulse 
output is updated, and generally there is a positive delay of at most few milliseconds between the two. At certain times (e.g. at approximately 25ms in fig. 6), the pulse output value appears to lead the processor value. This is attributable to uncertainty in the time synchronization between the two signals, which are collected on separate computer systems.

Several references have been made earlier in the paper to a measurement correction technique. This is a heuristic described in [5] which applies a correction to the phase difference proportional to the relative rate of change of amplitude of the sensor voltage signals. The empirical values of the coefficients describing the proportionality were obtained from data in which the dominant factor causing the rate of change of sensor signal amplitude was the presence in the signals of small components at the lower Coriolis frequency (the meter is not driven in the fundamental mode and thus has both an upper Coriolis frequency and a lower Coriolis frequency). There are suggestions that the effectiveness of the correction might be different when the sensor signals were contaminated by components at other frequencies. While the correction has been effective in reducing the phase noise in the majority of tests to date, including those where the noise was due to external vibrations, it requires the calculation of sensor voltage amplitude for the half-cycle after the one to which the correction is being applied. Thus there is a half-cycle delay in generating the corrected measurement. This contributed $6 \mathrm{~ms}$ to the overall $16 \mathrm{~ms}$ delay.

Figures 8 and 9 demonstrate the utility of the rate of change of amplitude correction technique in the same two experiments. In each figure three plots are given. The upper graphs (figs $8 \mathrm{a}$ and $8 \mathrm{~b}$ ) show the raw phase difference, as calculated off-line by Brunel using the sensor data captured at $500 \mathrm{kHz}$ during the experiments. The Brunel calculation is a 'baseline' phase difference value without any correction applied. Updates are provided four times per cycle and, being an off-line calculation, there is no time delay associated with the values thus calculated. The Brunel data independently verifies that the phase noise observed in the transmitter value (middle graphs, figs. $8 \mathrm{~b}$ and $9 \mathrm{~b}$ ) is present in the raw sensor signals, and is not merely the product of poor or faulty data processing. The lower graphs (figs. 8c and 9c) show the same transmitter value with the correction applied. Note that there remains some residual phase difference noise in the corrected measurement in the aftermath of the step changes, particularly the fast drop (compare figs. 4 and 5), and that the magnetic flowmeter does not show such flow variations.

Both Figure 8 and Figure 9 suggest that the response of the meter is in two parts, with the output reflecting the basic change in flow rate very rapidly, but thereafter the output shows an increased level of noise. Spectral analysis of the raw sensor signals suggests that much of this noise arises from the excitation of the Coriolis mode by the occurrence of the step. Clearly, if the output of the meter was damped sufficiently to remove the influence of this noise the effective response of the meter would be much slower. Considering the dynamic response on the meter, it is suggested that most users would prefer the corrected measurement (figs. 8c and 9c), with its improved precision, and an associated $16 \mathrm{~ms}$ delay, rather than the uncorrected measurement (figs. 8b and 9b) even with a delay of only $10 \mathrm{~ms}$.

Finally, a comparison is given between the results for the prototype meter and those reported by Wilkund and Peluso [1], who carried out step response tests on a variety of flow meter technologies (differential pressure sensor + orifice plate, electromagnetic flowmeter, vortex flowmeter, and one example of a coriolis meter). They modeled each flowmeter performance as either a first or second order response and quantified the response parameters for several meters of each type. Figure 10 shows the resulting step response for the fastest flowmeter of each class reported in [1], together with that of the prototype meter. Figure 10 suggests the prototype provides improved step response compared with current commercial meters.

A more detailed analysis of the issues surrounding the correction technique will be the subject of a further joint paper. 


\section{References}

[1] Wiklund, D. and M. Peluso (2002). Quantifying and specifying the dynamic response of flowmeters. ISA 2002 Technical Conference, Chicago.

[2] Wiklund, D. and M. Peluso (2002). Reducing process variability by using faster responding flowmeters in flow control. ISA 2002 Technical Conference, Chicago.

[3] Henry, M. P. and D.W. Clarke (1993). The Self-Validating Sensor: Rationale, Definitions and Examples. Control Engineering Practice, 1 (4), pp.585-610.

[4] BSI (2001). Specification for data quality metrics or industrial measurement and control systems, BS7986:2001. British Standards Institute, 389 Chiswick High Rd London W4 4AL.

[5] Henry, M. P, Clarke, D. W., Archer, N., Bowles, J., Leahy, M. J., Liu, R. P., Vignos, J., Zhou, F.B. (2000). A self-validating digital coriolis mass-flow meter: an overview. Control Engineering Practice, 8(5), pp. 487-506.

[6] Henry, M.P., Archer, N., Atia, M.R.A., Bowles, J., Clarke, D.W., Fraher, P.M.A., Page, I., Randall, G., and Yang, J.C-Y. (1996). Programmable hardware architectures for sensor validation. Control Engineering Practice, 4(10), 1339-1354.

[7] Celoxica (2002). http://www.celoxica.com/

[8] Zamora, M and M. P. Henry (2002). Generation of Frequency Output for Instrumentation Applications using Digital Hardware. Submitted to Sensor Review, August 2002. 


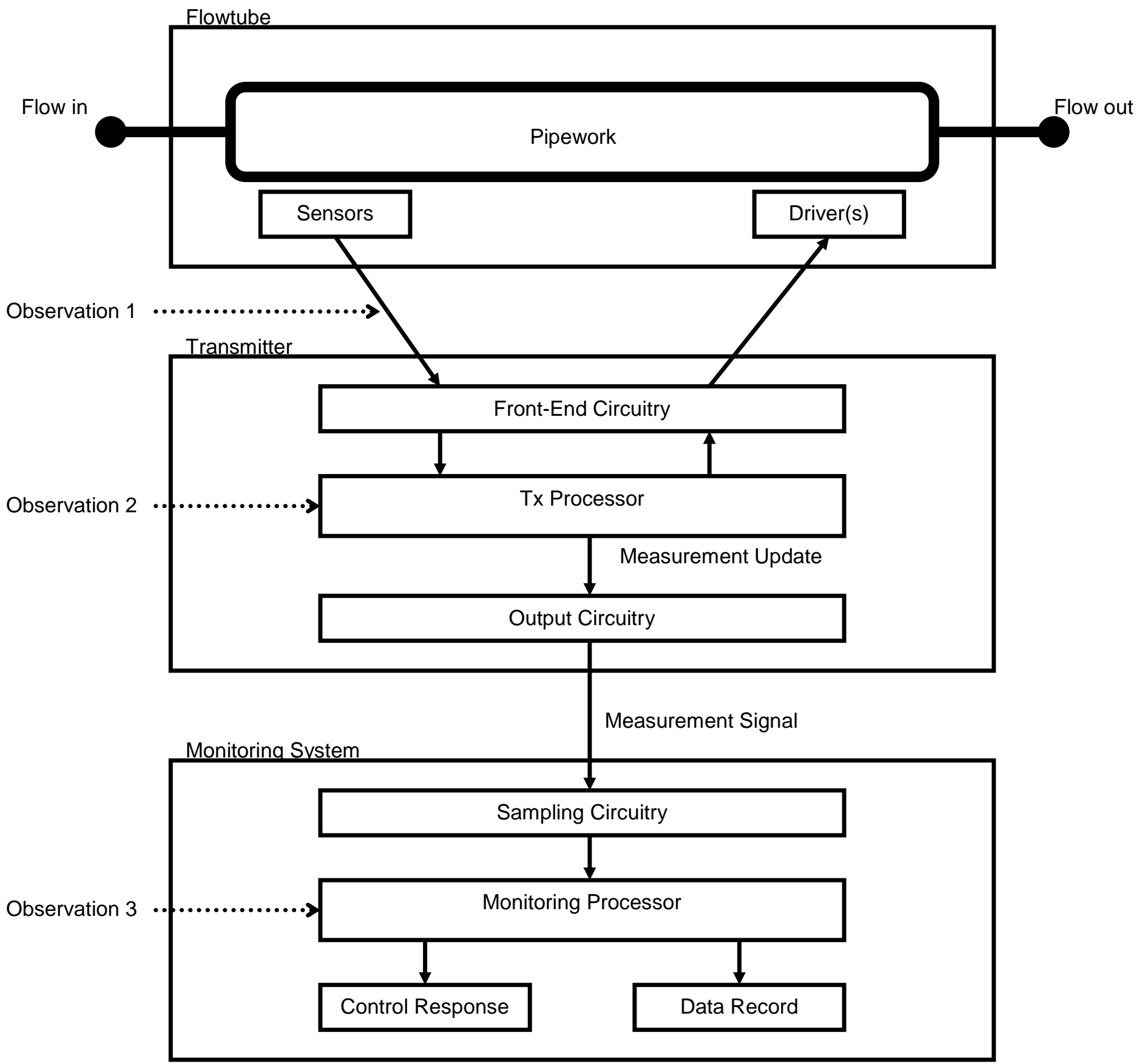

Figure 1. Meter Flowtube, Transmitter and Monitoring System 


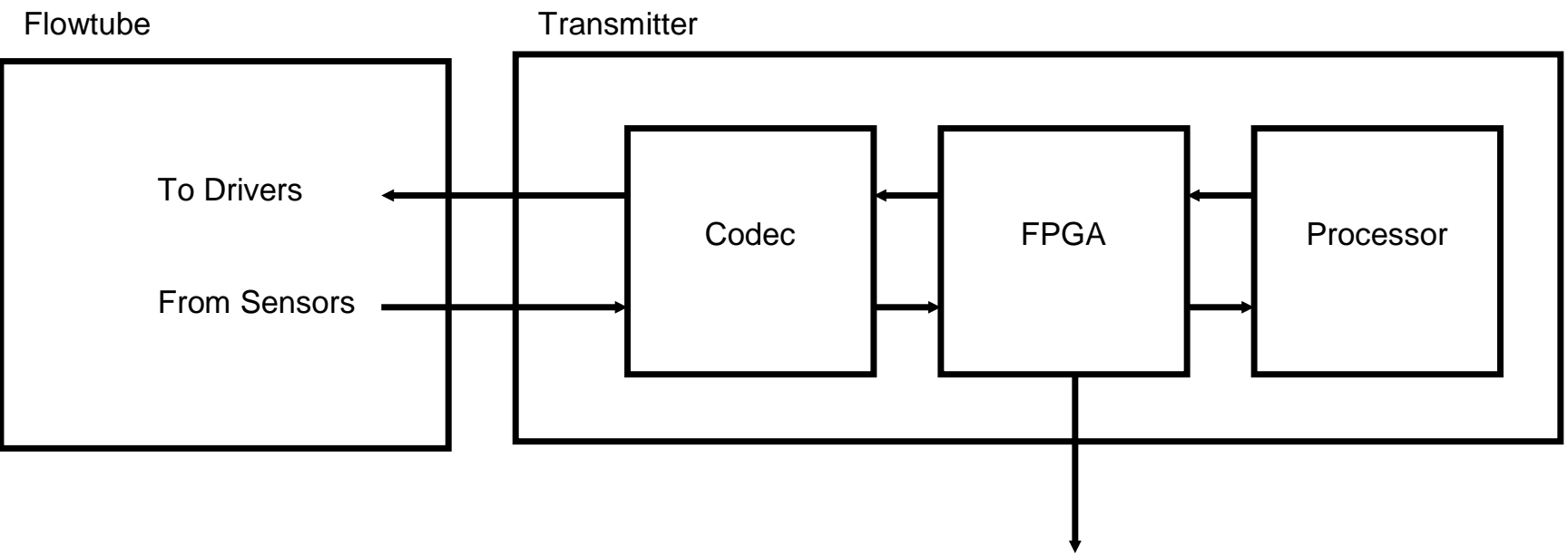

Pulse Output

Figure 2. Prototype Transmitter Architecture 


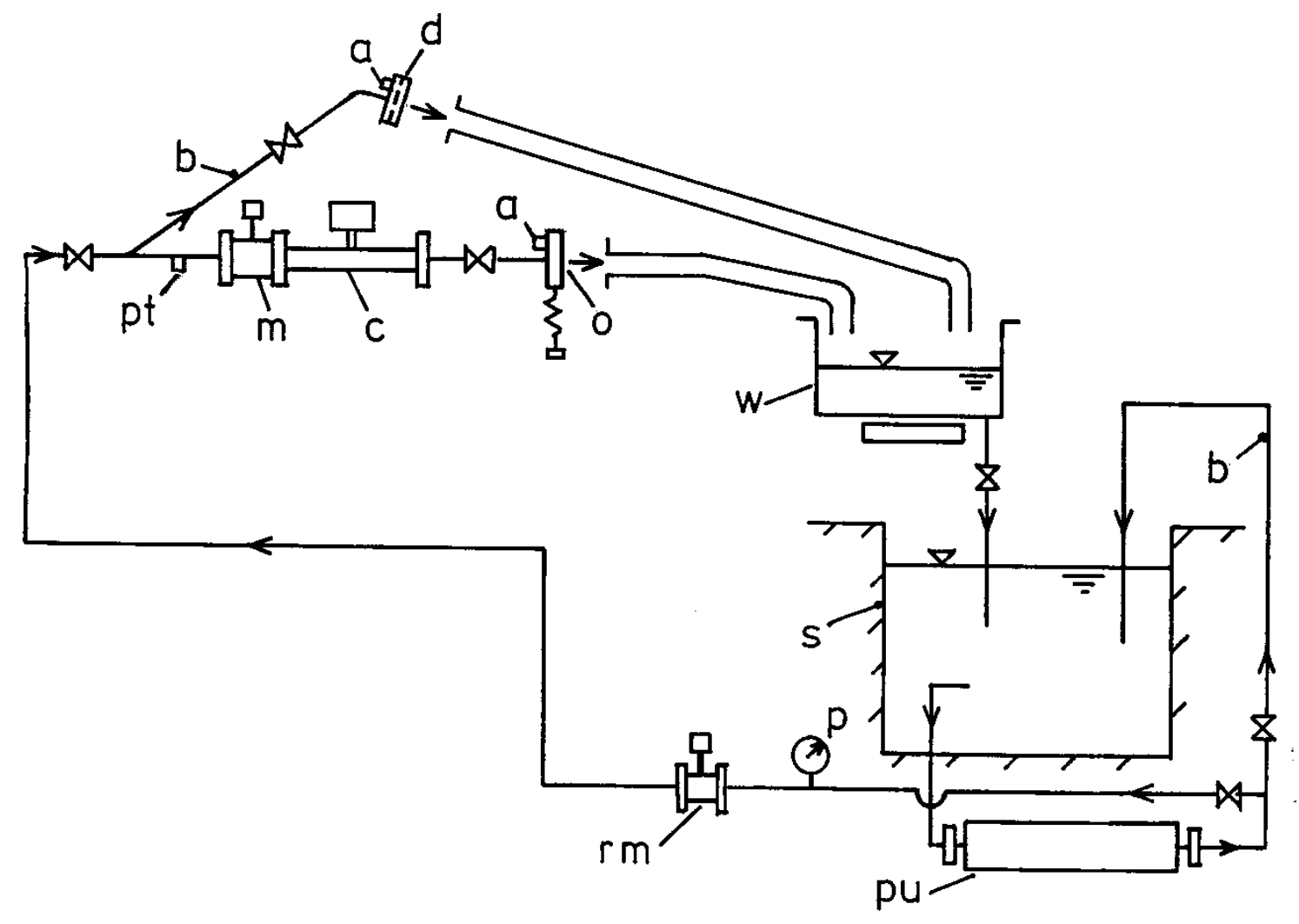

$\begin{array}{llllll}\mathrm{a} & \text { accelerometer } & \mathrm{b} & \text { by-pass } & \mathrm{c} & \text { Coriolis test meter } \\ \mathrm{d} & \text { burstable diaphragm } & \mathrm{m} & \text { electromagnetic flowmeter } & \mathrm{o} & \text { variable area orifice } \\ \mathrm{p} & \text { pressure gauge } & \mathrm{pt} & \text { pressure transducer } & \mathrm{pu} & \text { pump } \\ \text { rm } & \text { reference flowmeter } & \mathrm{s} & \text { sump } & \mathrm{w} & \text { weigh-tank }\end{array}$

Figure 3. Brunel Flow-rig. 


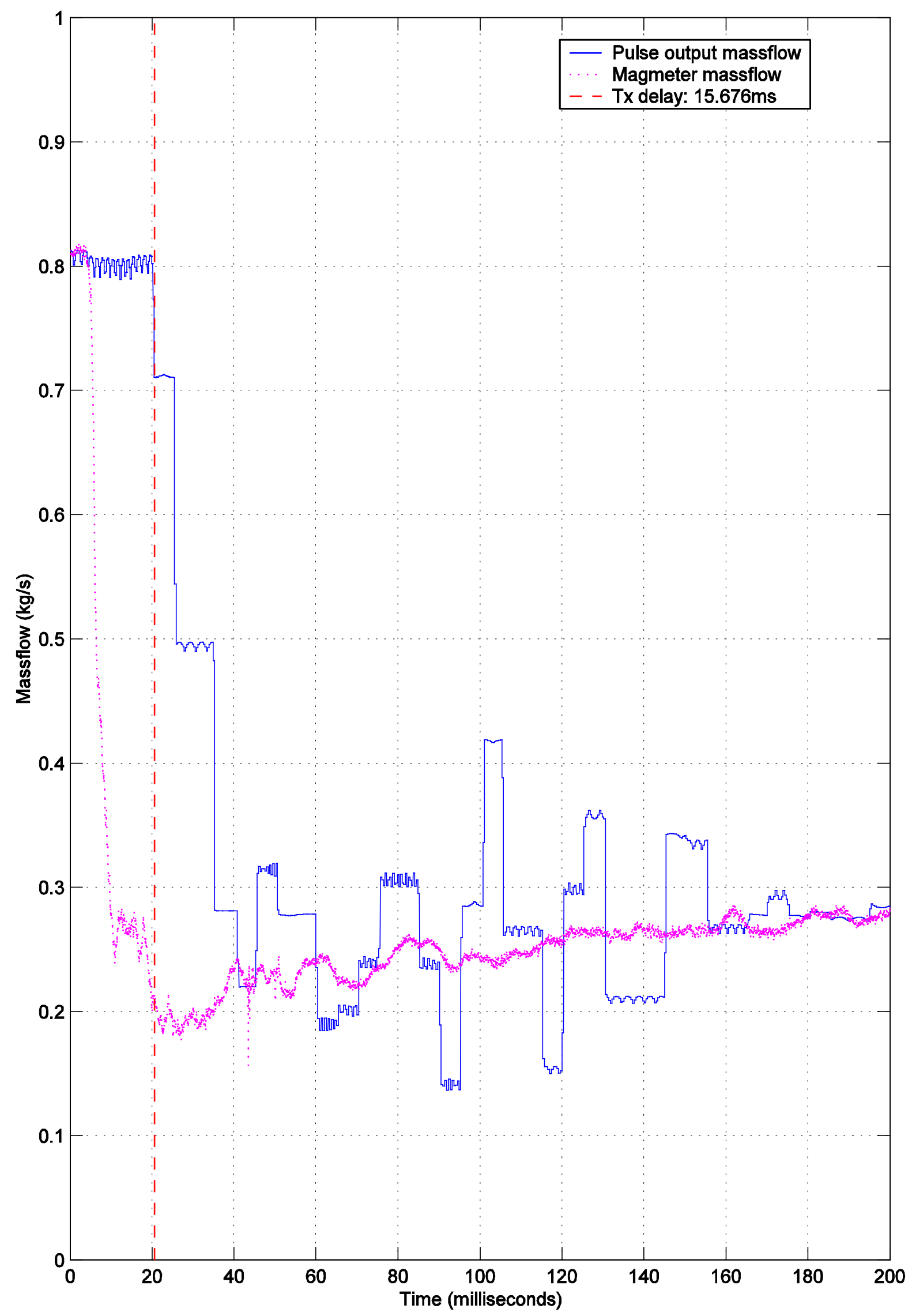

Figure 4. Pulse output after a rapid drop in massflow, with the magnetic flowmeter signal. 


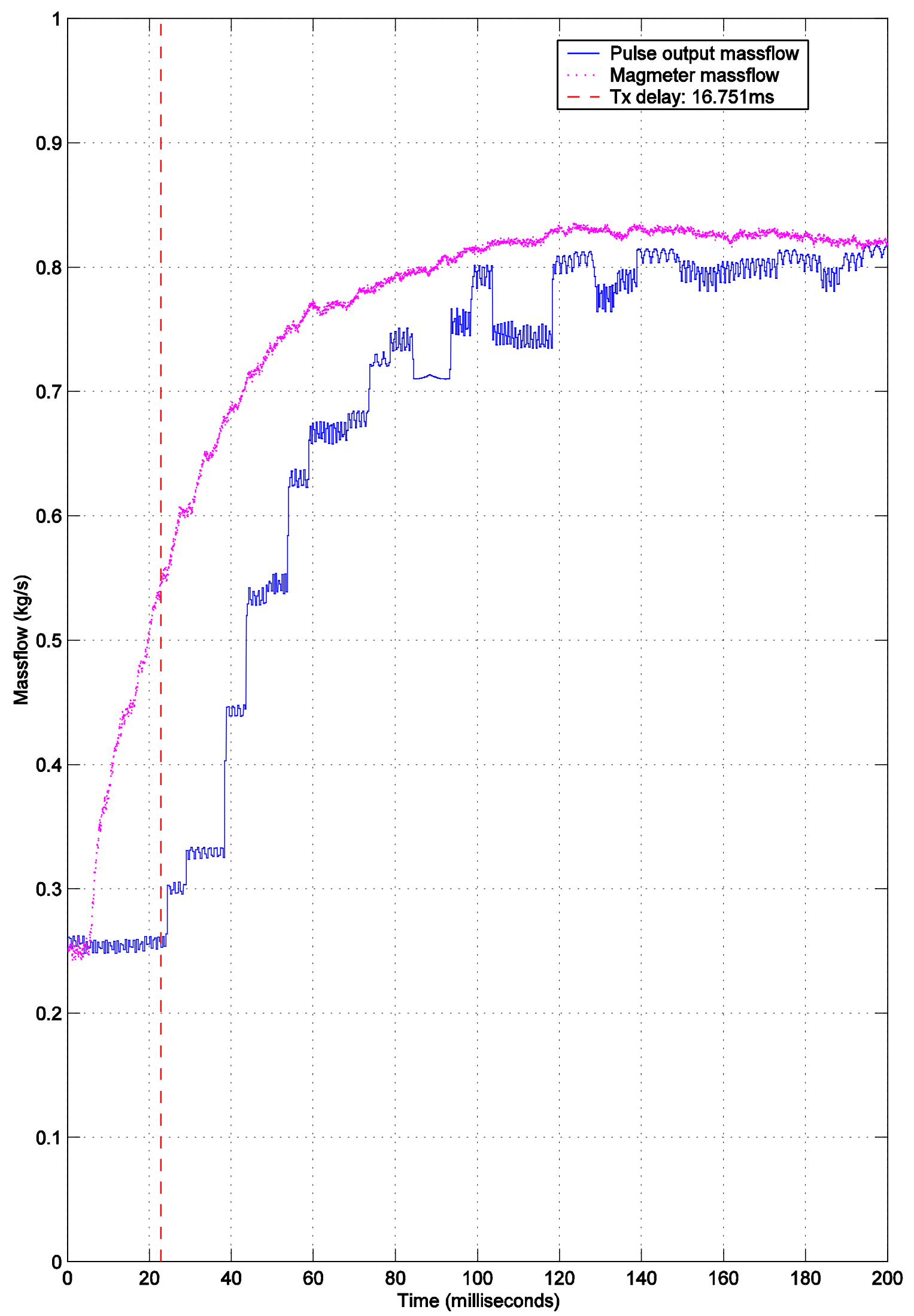

Figure 5. Pulse output after a rapid increase in massflow, with the magnetic flowmeter signal. 


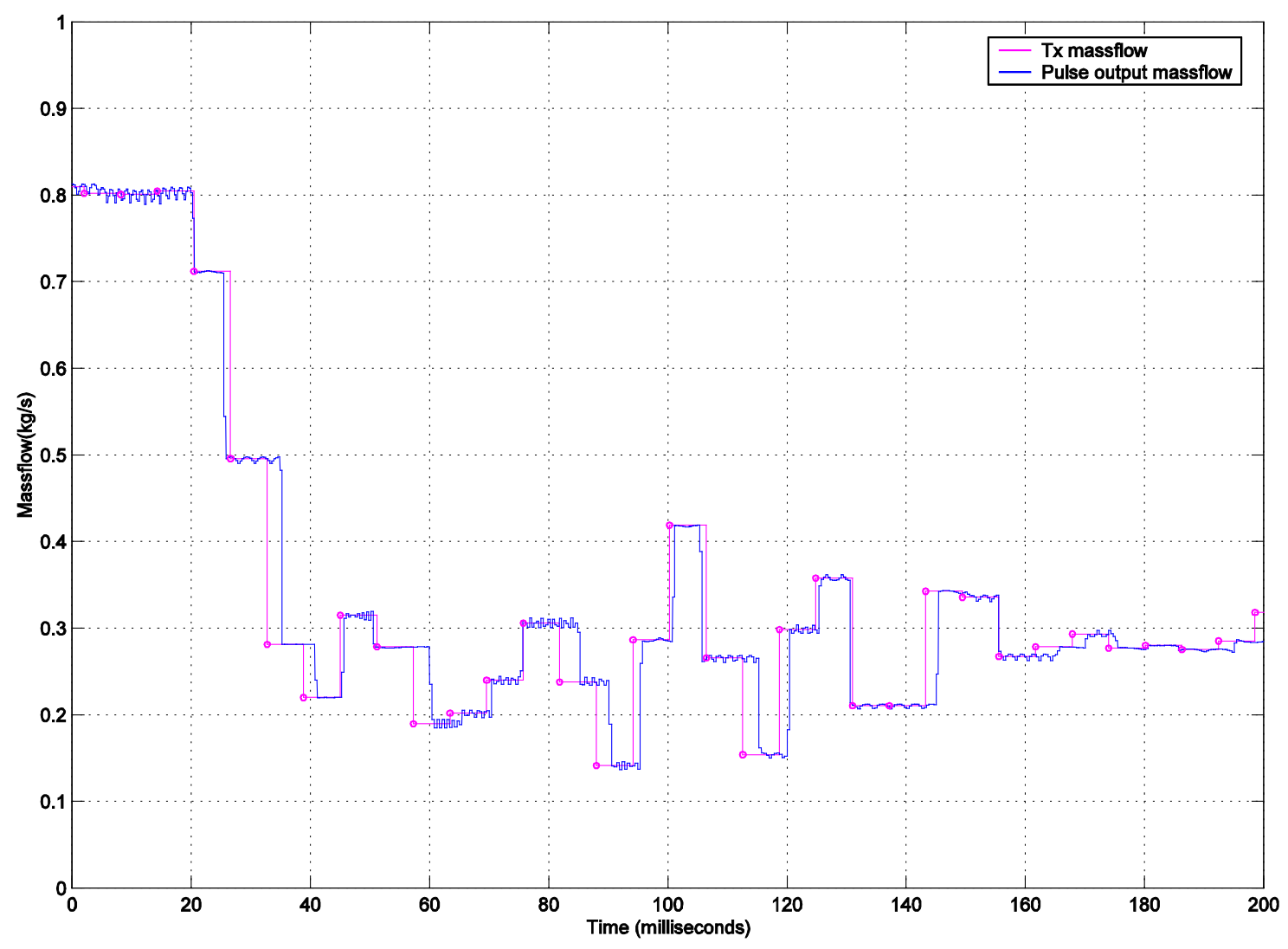

Figure 6. The transmitter massflow and pulse output values after a rapid decrease in massflow

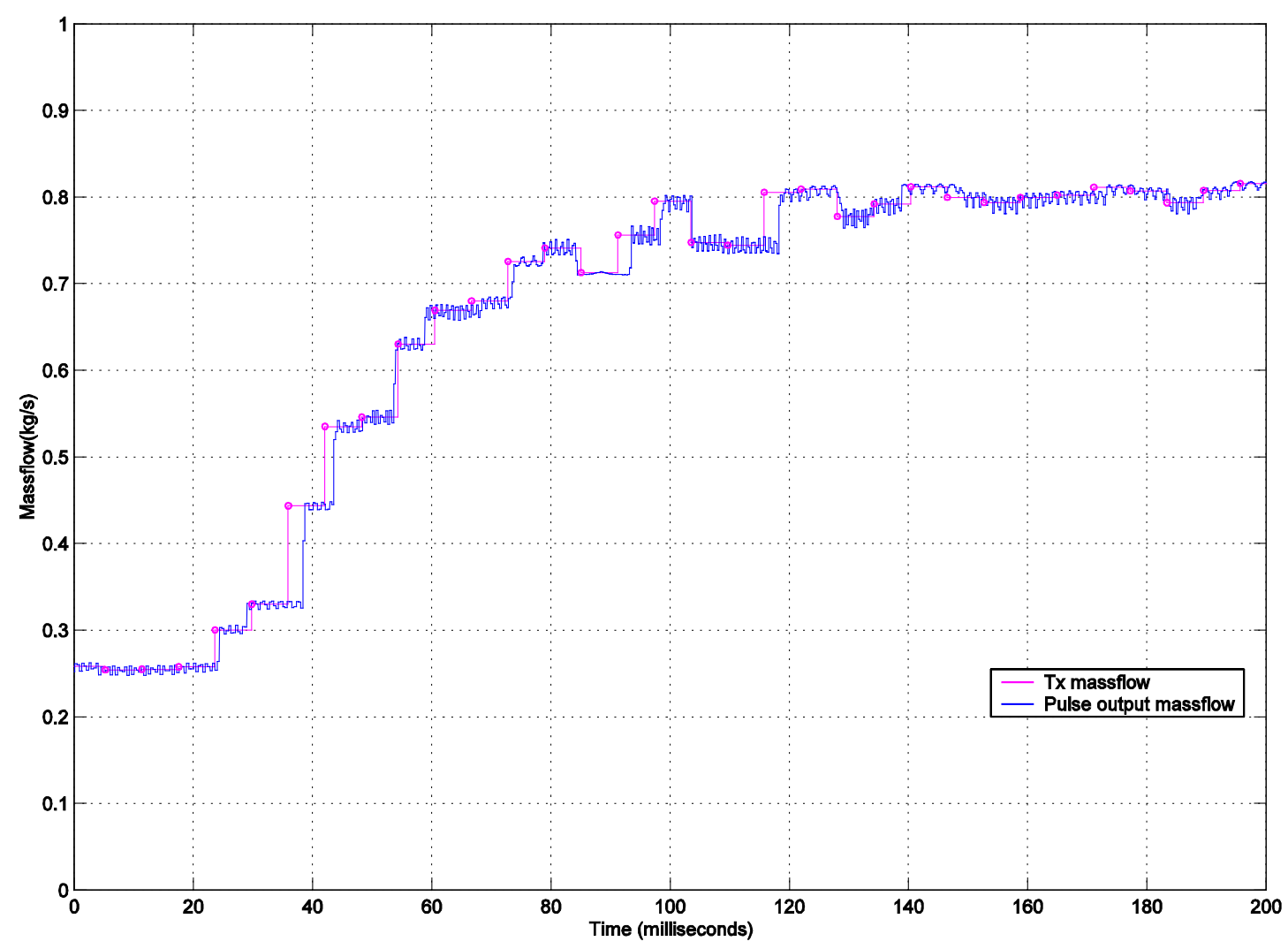

Figure 7. The transmitter massflow and pulse output values after a rapid increase in massflow 
(a) Raw phase difference from sensor data calculated off-line by Brunel

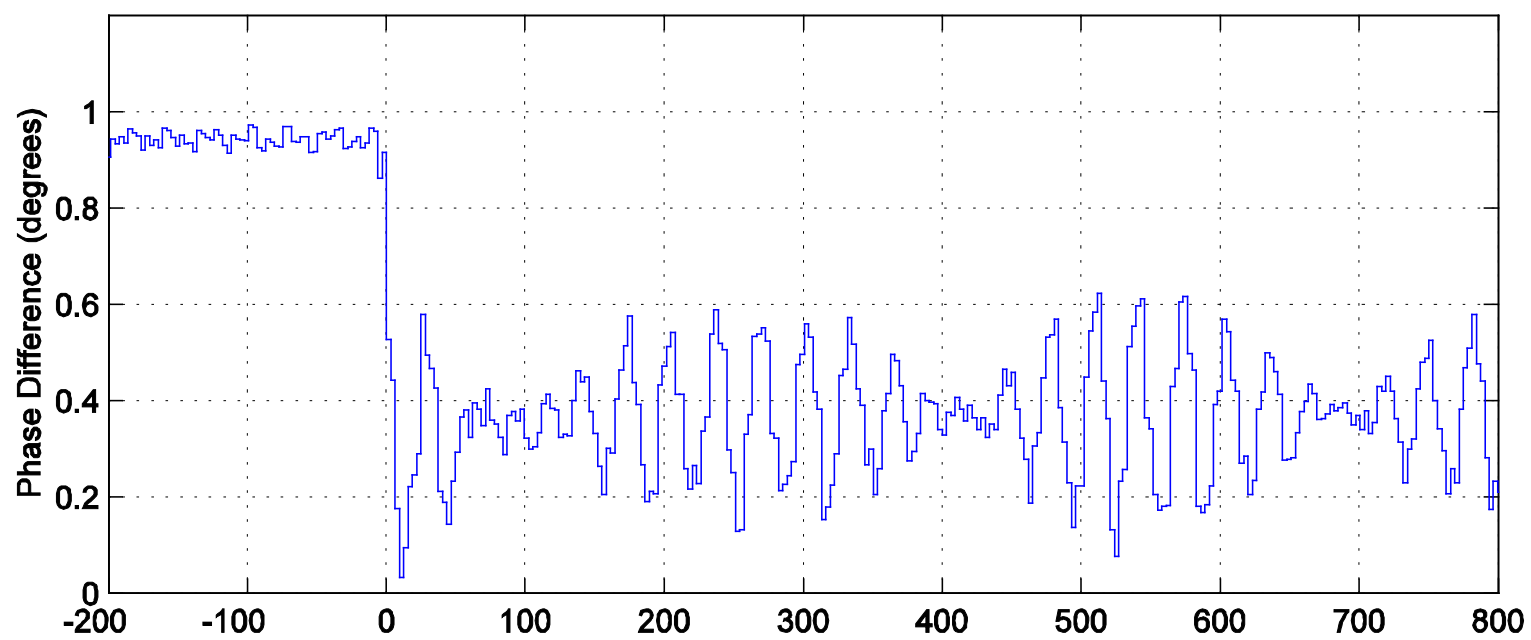

(b) Raw phase difference from prototype transmitter

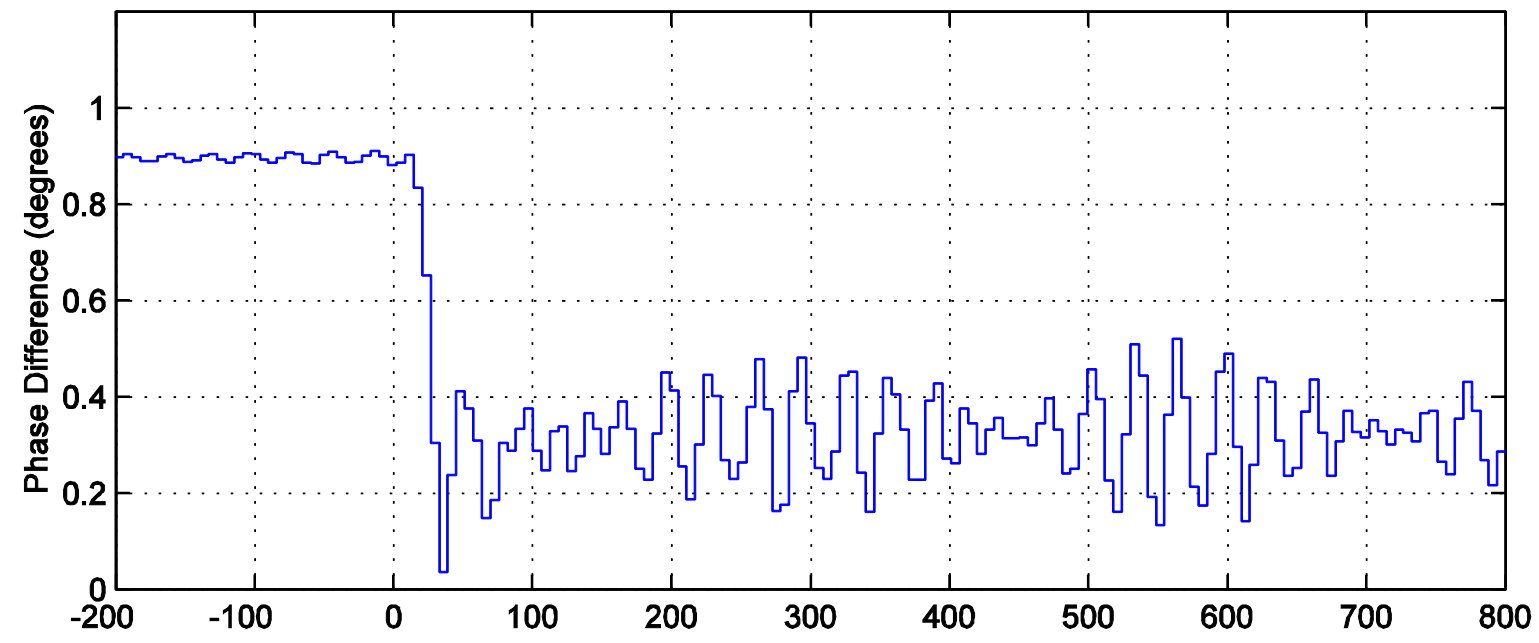

(c) Corrected phase difference from prototype transmitter

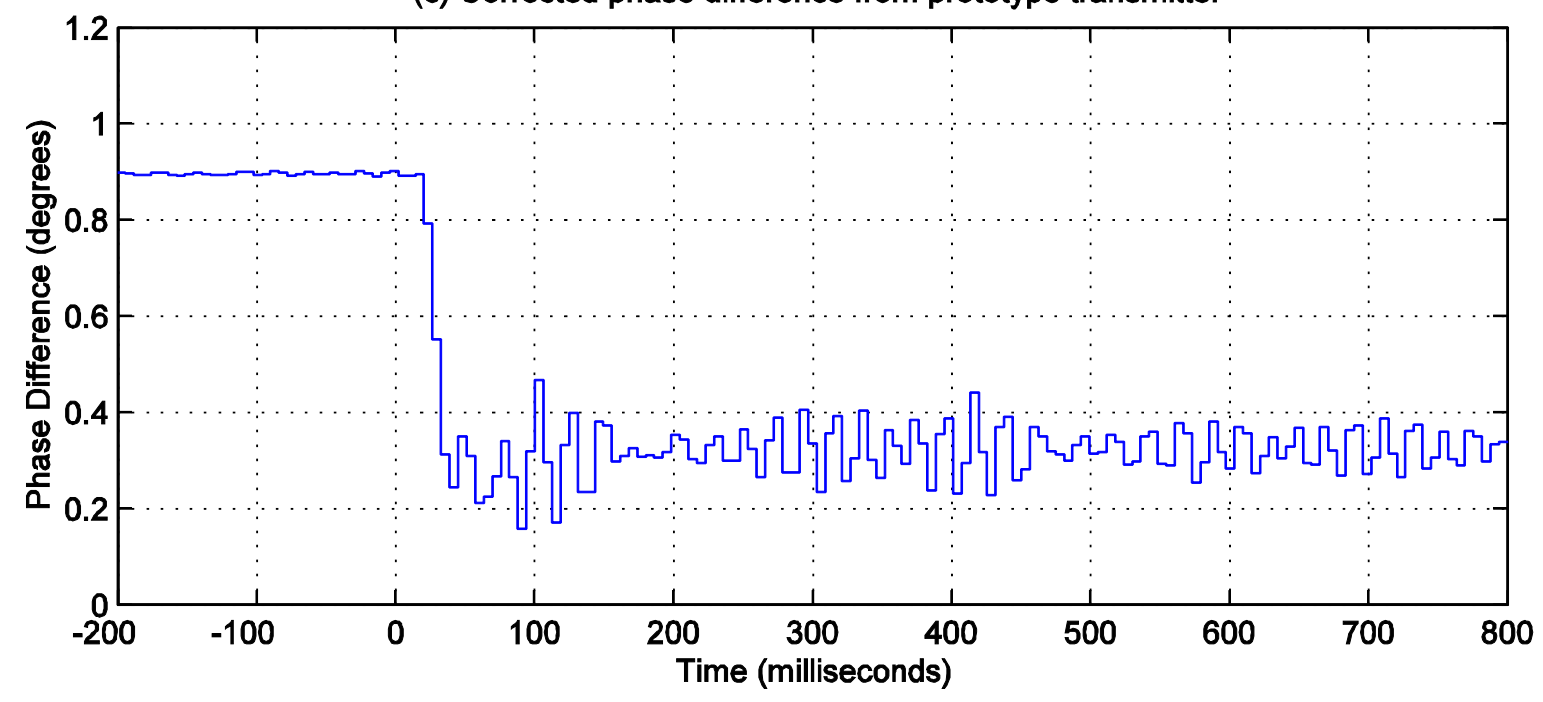

Figure 8. Raw and corrected phase difference during a drop in flowrate 
(a) Raw phase difference from sensor data calculated off-line by Brunel

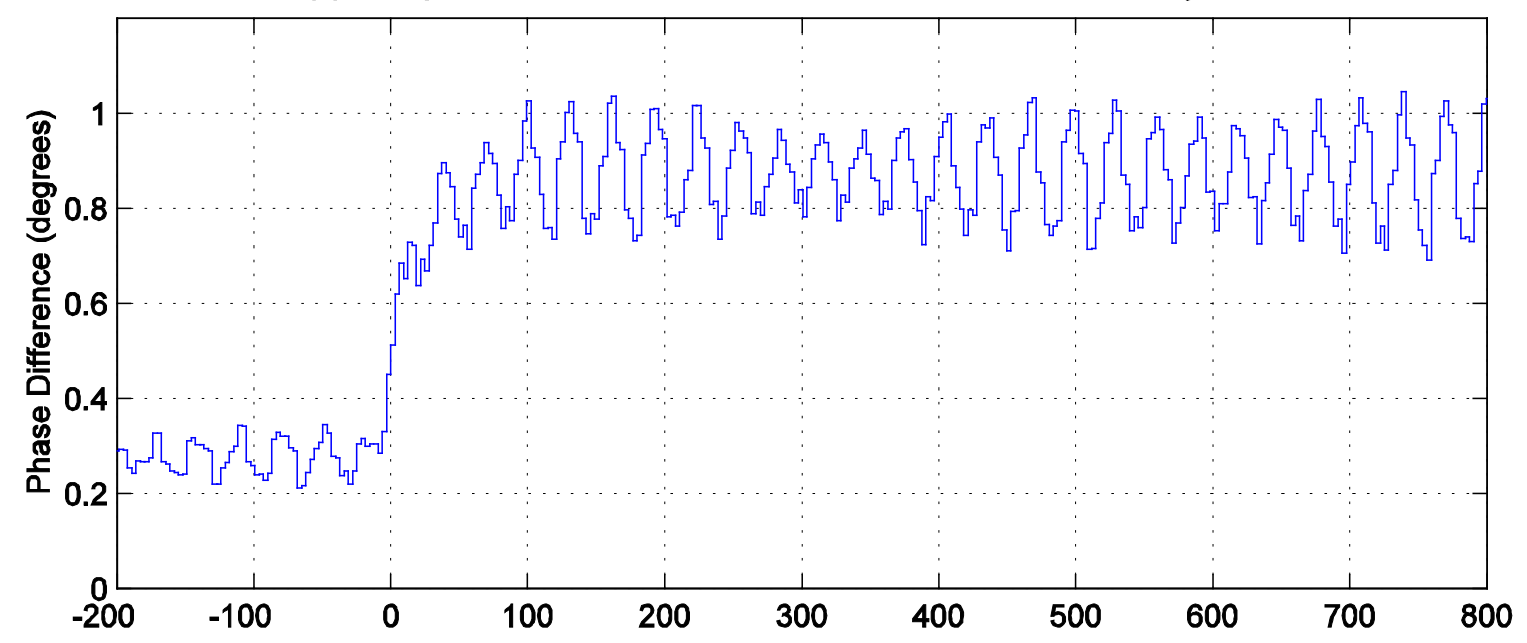

(b) Raw phase difference from prototype transmitter

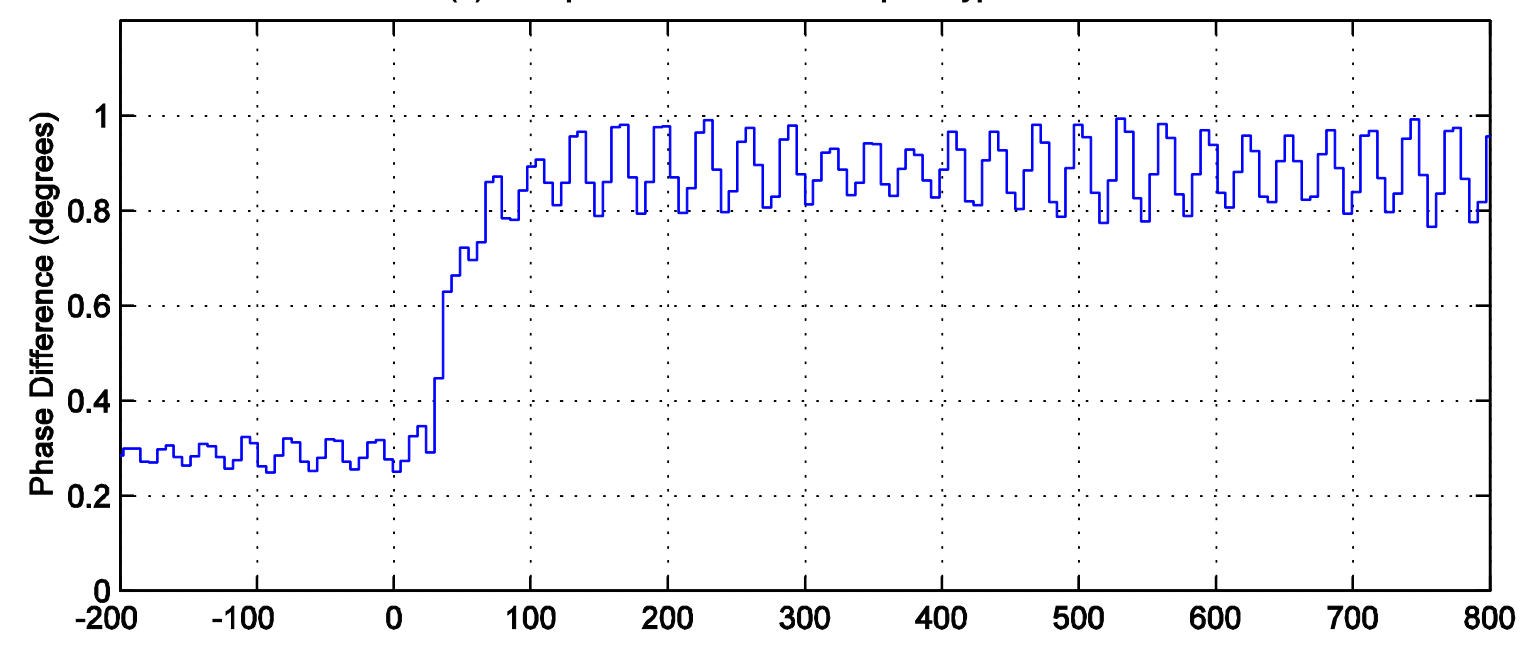

(c) Corrected phase difference from prototype transmitter

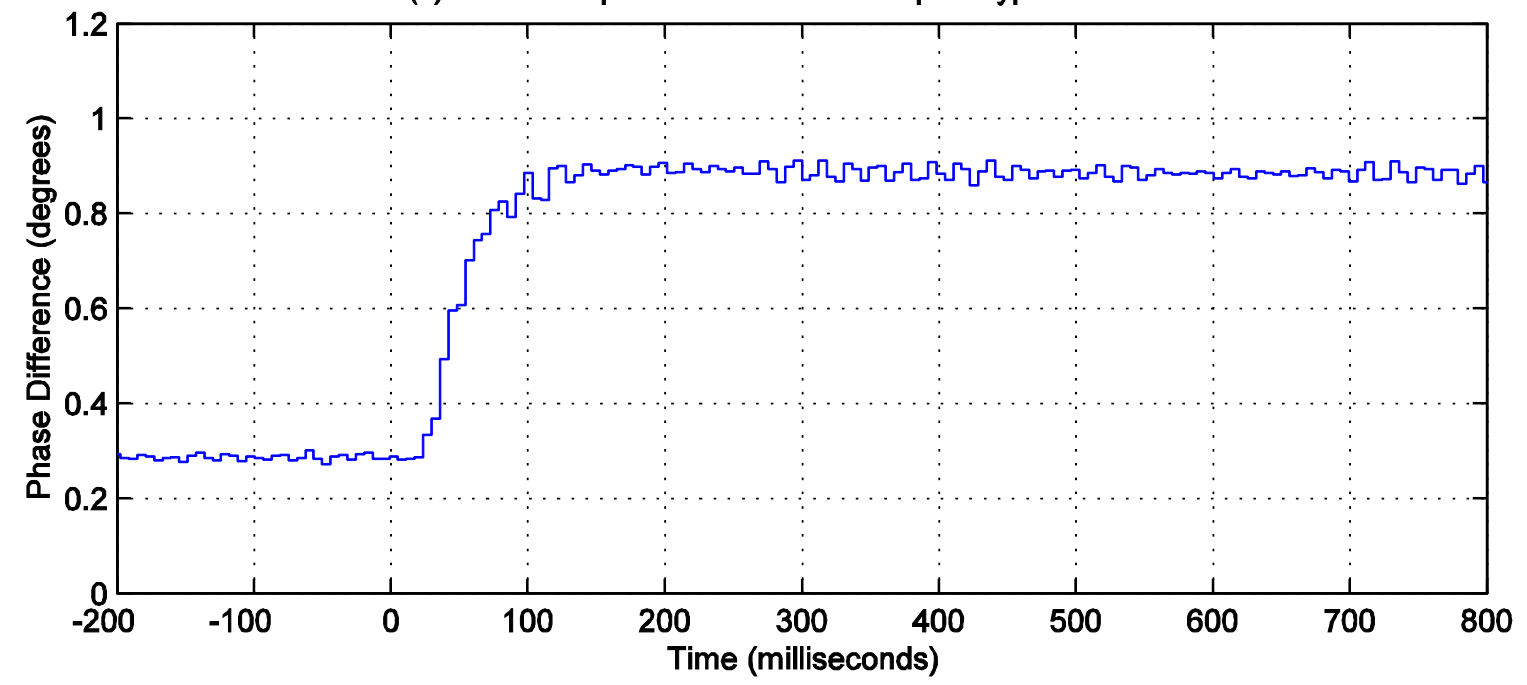

Figure 9. Raw and corrected phase difference during a rise in flowrate 


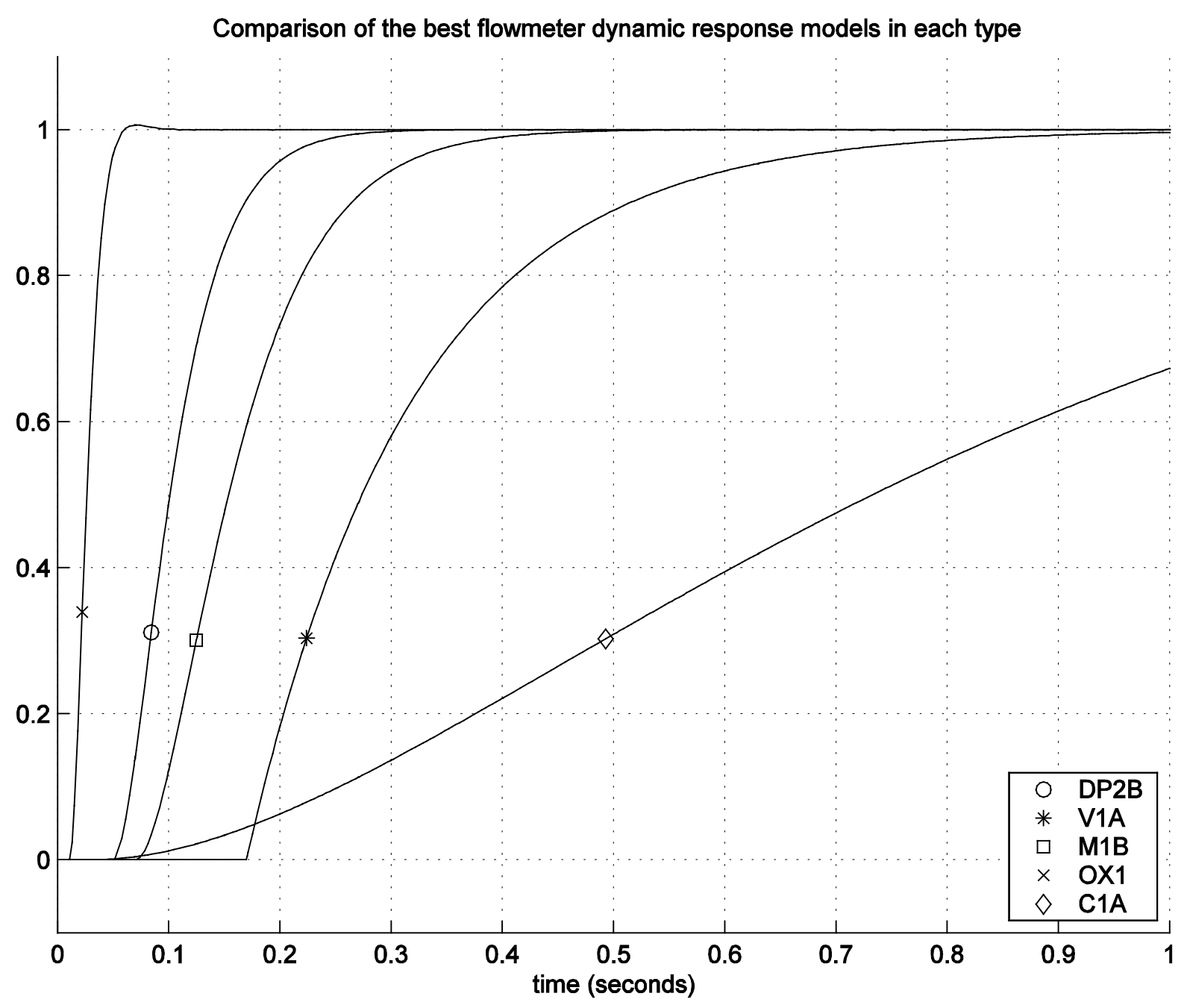

Figure 10. Fastest dynamic response of each type of flowmeter reported by Wilkund and Peluso (2002), together with Oxford prototype response (OX1), on a nominal $0 . .1$ scale. DP = Differential Pressure, $\mathrm{V}=$ Vortex Flow, $\mathrm{M}=$ Magnetic Flow, $\mathrm{C}$ = commercial coriolis massflow meter. 